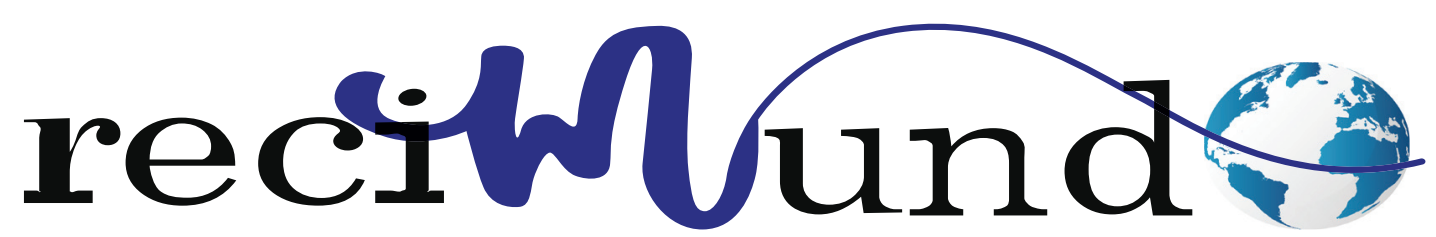

Revista Científica Mundo de la Investigación y el Conocimiento

DOI: $10.26820 /$ recimundo/4.(2).mayo.2020.16-30

URL: http://recimundo.com/index.php/es/article/view/819

EDITORIAL: Saberes del Conocimiento

REVISTA: RECIMUNDO

ISSN: 2588-073X

TIPO DE INVESTIGACIÓN: Artículo de Revisión

CÓDIGO UNESCO: 1203 Ciencia de Los Ordenadores; 1203.04 Inteligencia Artificial

PAGINAS: $16-30$

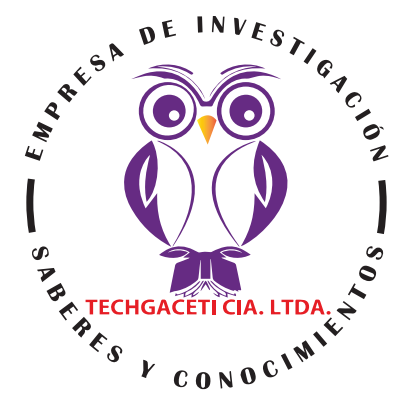

\title{
Inteligencia artificial, sistemas inteligentes, agentes inteligentes
}

Artificial intelligence, smart systems, smart agents

Inteligência artificial, sistemas inteligentes, agentes inteligentes

Jesús Antonio Coloma Garofalo; José Andrés Vargas Salazar²; Cristian Andres Sanaguano Guevara³;

Ángel Geovanny Rochina Chisag ${ }^{4}$

\section{RECIBIDO: 15/03/2020 ACEPTADO: 20/03/2020 PUBLICADO: 20/05/2020}

1. Master Universitario en Ingeniería de Software y Sistemas Informáticos; Ingeniero en Sistemas Computacionales; Universidad Estatal de Bolívar, Bolívar, Ecuador; jcoloma@ueb.edu.ec; iD https://orcid.org/0000-00031827-3296

2. Master Universitario en Ingeniería de Software y Sistemas Informáticos; Ingeniero en Sistemas Computacionales; Universidad Estatal de Bolívar, Bolívar, Ecuador; jandres1290@gmail.com; (D) https://orcid.org/0000-00029373-7276

3. Profesional en Formación; Universidad de las Fuerzas Armadas; Sangolquí, Ecuador; casanaguano@espe. edu.ec; (D) https://orcid.org/0000-0001-5578-5122

4. Ingeniero en Sistemas Computacionales; Investigador Independiente; Guayaquil, Ecuador; rochitheonly@ gmail.com; (iD https://orcid.org/0000-0002-1570-9624

\section{CORRESPONDENCIA \\ Jesús Antonio Coloma Garofalo \\ jcoloma@ueb.edu.ec \\ Bolívar, Ecuador}

(C) RECIMUNDO; Editorial Saberes del Conocimiento, 2020 


\section{RESUMEN}

En inteligencia artificial, un agente inteligente (IA) es una entidad autónoma que observa a través de sensores y actúa sobre un entorno utilizando actuadores (es decir, es un agente) y dirige su actividad hacia el logro de objetivos (es decir, es racional). Los agentes inteligentes también pueden aprender o usar el conocimiento para lograr sus objetivos. Los agentes inteligentes en inteligencia artificial están estrechamente relacionados con los agentes en economía, y las versiones del paradigma del agente inteligente se estudian en ciencias cognitivas, ética, filosofía de la razón práctica, así como en muchos modelos sociocognitivos interdisciplinarios y simulaciones sociales informáticas. Un sistema de múltiples agentes (MAS) es un sistema compuesto por múltiples agentes inteligentes que interactúan. Los sistemas de múltiples agentes se pueden usar para resolver problemas que son difíciles o imposibles de resolver para un agente individual o un sistema monolítico. Los sistemas de múltiples agentes consisten en agentes y su entorno. Por lo general, la investigación de sistemas de múltiples agentes se refiere a agentes de software. Sin embargo, los agentes en un sistema de múltiples agentes también podrían ser robots, humanos o equipos humanos. Un sistema de múltiples agentes puede contener equipos combinados de agentes humanos. Este trabajo presenta una breve distribución sobre los sistemas de agente inteligente y agente múltiple.

Palabras clave: Inteligencia artificial, Agentes inteligentes, Sistemas múltiples de agentes.

\section{ABSTRACT}

In artificial intelligence, an intelligent agent (AI) is an autonomous entity that observes through sensors and acts on an environment using actuators (that is, is an agent) and directs its activity towards the achievement of objectives (that is, it is rational ). Smart agents can also learn or use knowledge to achieve their goals. Intelligent agents in artificial intelligence are closely related to agents in economics, and versions of the intelligent agent paradigm are studied in cognitive science, ethics, the philosophy of practical reason, as well as in many interdisciplinary sociocognitive models and computer social simulations. A multiple agent system (MAS) is a system made up of multiple intelligent agents that interact. Multiple agent systems can be used to solve problems that are difficult or impossible to solve for an individual agent or monolithic system. Multi-agent systems consist of agents and their environment. In general, the investigation of systems of multiple agents refers to software agents. However, agents in a multi-agent system could also be robots, humans, or human teams. A multiple agent system can contain combined teams of human agents. This work presents a brief distribution on the intelligent agent and multiple agent systems.

Keywords: Artificial intelligence, Intelligent agents, Multiple agent systems.

\section{RESUMO}

Na inteligência artificial, um agente inteligente (IA) é uma entidade autônoma que observa através de sensores e atua em um ambiente usando atuadores (isto é, é um agente) e direciona sua atividade para a consecução de objetivos (ou seja, é racional). Os agentes inteligentes também podem aprender ou usar o conhecimento para atingir seus objetivos. Os agentes inteligentes em inteligência artificial estão intimamente relacionados aos agentes da economia, e as versões do paradigma do agente inteligente são estudadas em ciências cognitivas, ética, filosofia da razão prática, bem como em muitos modelos sociocognitivos interdisciplinares e simulações sociais em computador. Um sistema de múltiplos agentes (MAS) é um sistema composto por vários agentes inteligentes que interagem. Vários sistemas de agentes podem ser usados para resolver problemas difíceis ou impossíveis de resolver para um agente individual ou sistema monolítico. Os sistemas multi-agente consistem em agentes e seu ambiente. Em geral, a investigação de sistemas de múltiplos agentes refere-se a agentes de software. No entanto, os agentes em um sistema multiagente também podem ser robôs, humanos ou equipes humanas. Um sistema de múltiplos agentes pode conter equipes combinadas de agentes humanos. Este trabalho apresenta uma breve distribuição nos sistemas de agentes inteligentes e múltiplos agentes.

Palavras-chave: Inteligência artificial, Agentes inteligentes, Sistemas de múltiplos agentes. 


\section{Introducción}

Aunque no hay consenso sobre la definición de un agente, parece estar generalmente de acuerdo en que un agente se encuentra dentro de un entorno donde es capaz de actuar (utilizando efectores), y cuyas acciones pueden causar cambios en el entorno. También se supone que el agente puede detectar cambios en su entorno (mediante sensores) (Boella, Damiano, \& Lesmo, 2009).

Otro requisito a menudo asumido sobre un agente es el de la habilidad social. Es decir, se supone que un agente puede comunicarse y coordinar sus acciones con las de otros agentes ubicados en el entorno del agente. Se supone que las acciones Ilevadas a cabo por un agente son el resultado de decisiones tomadas por el agente basadas en lo que siente en su entorno y sobre la meta que el agente está tratando de lograr. Dichas decisiones de acción deben ser más inteligentes. Dicha inteligencia podría obtenerse como resultado del conocimiento incorporado por las reglas en una base de conocimiento o en alguna otra forma de esquema de representación del conocimiento.

Recientemente, el Sistema de múltiples agentes (MAS) se ha convertido en uno de los temas dominantes de investigación en Inteligencia Artificial (IA). Esta parte se está separando gradualmente de la inteligencia artificial distribuida como una disciplina independiente, basada en resultados de investigación tanto en otras partes de la IA como en el área de la informática.

El sistema de múltiples agentes es una colección de agentes que cooperan entre sí para cumplir objetivos comunes e individuales (en algunos entornos también pueden competir). En el sistema de múltiples agentes, los diferentes agentes a menudo tienen diferentes roles y objetivos individuales (Elmahalawy, 2011).
Un problema para el sistema de múltiples agentes es cómo puede mantener la coherencia global, es decir, comportarse bien como un sistema sin un control global explícito. Es útil organizarlos en alguna forma de estructura. En una estructura jerárquica, algunos agentes están diseñados para detectar y gestionar las interdependencias entre las acciones de otros agentes. Este agente puede tener poder de autoridad sobre otros agentes y, por lo tanto, puede controlar, por ejemplo, los objetivos de otros agentes. Esta estructura puede contrastarse con la descentralizada donde no existen tales agentes de autoridad.

El sistema multiagente es el paradigma ampliamente utilizado para el modelado, planificación y control de varios procesos. En general, utiliza técnicas de negociación distribuidas para lograr objetivos particulares. Además de la planificación centralizada estándar y los mecanismos de optimización, el sistema de múltiples agentes admite la replanificación local con cambios mínimos necesarios de todo el plan.

El área de investigación de la Simulación basada en agentes (ABS) continúa produciendo técnicas, herramientas y métodos. Además, se han desarrollado una gran cantidad de aplicaciones de ABS. Por aplicación de simulación basada en agente nos referimos a simulaciones informáticas reales basadas en el modelado basado en agente de un sistema real (o imaginado) para resolver un problema concreto. Una aplicación de simulación basada en agente modela y simula un sistema real que consiste en un conjunto de entidades. La simulación basada en agente en sí misma puede verse como un sistema de múltiples agentes compuesto por un conjunto de agentes (software). Es decir, existe una correspondencia entre el sistema real y el sistema de múltiples agentes, así como entre las entidades (reales) y los agentes. 


\section{Metodología}

Para el desarrollo de este proceso investigativo, se plantea como metodología la encaminada hacia una orientación científica particular que se encuentra determinada por la necesidad de indagar en forma precisa y coherente una situación, en tal sentido (Davila, 2015) define la metodología "como aquellos pasos previos que son seleccionados por el investigador para lograr resultados favorables que le ayuden a plantear nuevas ideas".(p.66)

Lo citado por el autor, lleva a entender que el desarrollo de la acción investigativa busca simplemente coordinar acciones enmarcadas en una revisión bibliográfica con el fin de complementar ideas previas relacionadas a la inteligencia artificial y agentes múltiples de inteligencia a través de una revisión de literatura, para así finalmente elaborar un cuerpo de consideraciones generales que ayuden a ampliar el interés propuesto.

\section{Tipo de Investigación}

Dentro de toda práctica investigativa, se precisan acciones de carácter metodológico mediante las cuales, se logra conocer y proyectar los eventos posibles que la determinan, así como las características que hacen del acto científico un proceso interactivo ajustado a una realidad posible de ser interpretada. En este sentido, se puede decir, que la presente investigación corresponde al tipo documental, definido por Castro (2016), "se ocupa del estudio de problemas planteados a nivel teórico, la información requerida para abordarlos se encuentra básicamente en materiales impresos, audiovisuales y /o electrónicos". (p.41).

En consideración a esta definición, la orientación metodológica permitió la oportunidad de cumplir con una serie de actividades inherentes a la revisión y lectura de diversos documentos donde se encontraron ideas explicitas relacionadas con los tópicos en- cargados de identificar a cada característica insertada en el estudio. Por lo tanto, se realizaron continuas interpretaciones con el claro propósito de revisar aquellas apreciaciones o investigaciones propuestas por diferentes investigadores relacionadas con el tema de interés, para luego dar la respectiva argumentación a los planteamientos, en función a las necesidades encontradas en la indagación.

\section{Fuentes Documentales}

El análisis correspondiente a las características que predomina en el tema seleccionado, llevan a incluir diferentes fuentes documentales encargadas de darle el respectivo apoyo y en ese sentido cumplir con la valoración de los hechos a fin de generar nuevos criterios que sirven de referencia a otros procesos investigativos. Para (CASTRO, 2016) las fuentes documentales incorporadas en la investigación documental o bibliográfica, "representa la suma de materiales sistemáticos que son revisados en forma rigurosa y profunda para llegar a un análisis del fenómeno".(p.41). Por lo tanto, se procedió a cumplir con la realización de una lectura previa determinada para encontrar aquellos aspectos estrechamente vinculados con el tema, con el fin de explicar mediante un desarrollo las respectivas apreciaciones generales de importancia.

\section{Técnicas para la Recolección de la Infor- mación}

La conducción de la investigación para ser realizada en función a las particularidades que determinan a los estudios documentales, tiene como fin el desarrollo de un conjunto de acciones encargadas de llevar a la selección de técnicas estrechamente vinculadas con las características del estudio. En tal sentido, (Bolívar, 2015), refiere, que es "una técnica particular para aportar ayuda a los procedimientos de selección de las ideas primarias y secundarias". (p. 71). 
Por ello, se procedió a la utilización del subrayado, resúmenes, fichaje, como parte básica para la revisión y selección de los documentos que presentan el contenido teórico. Es decir, que mediante la aplicación de estas técnicas se pudo llegar a recoger informaciones en cuanto a la revisión bibliográfica de los diversos elementos encargados de orientar el proceso de investigación. Tal como lo expresa, (Bolívar, 2015) "las técnicas documentales proporcionan las herramientas esenciales y determinantes para responder a los objetivos formulados y llegar a resultados efectivos" (p. 58). Es decir, para responder con eficiencia a las necesidades investigativas, se introdujeron como técnica de recolección el método inductivo, que hizo posible llevar a cabo una valoración de los hechos de forma particular para llegar a la explicación desde una visión general.

Asimismo, se emplearon las técnicas de análisis de información para la realización de la investigación que fue ejecutada bajo la dinámica de aplicar diversos elementos encargados de determinar el camino a recorrer por el estudio, según, (Bolívar, 2015) las técnicas de procesamiento de datos en los estudios documentales "son las encargadas de ofrecer al investigador la visión o pasos que debe cumplir durante su ejercicio, cada una de ellas debe estar en correspondencia con el nivel a emplear" (p. 123). Esto indica, que para llevar a cabo el procesamiento de los datos obtenidos una vez aplicado las técnicas seleccionadas, tales como: fichas de resumen, textual, registros descriptivos entre otros, los mismos se deben ajustar al nivel que ha sido seleccionado.

\section{Resultados}

La tecnología de agente ha recibido una gran atención en los últimos años y, como resultado, la industria está comenzando a interesarse en usar esta tecnología para desarrollar sus propios productos.
Un Agente inteligente (IA) es una entidad de software que realiza algunas operaciones en nombre de un usuario u otro programa con cierto grado de independencia o autonomía, y al hacerlo, emplea cierto conocimiento o representación de los objetivos o deseos del usuario.

Las tecnologías de agentes provienen del campo de la inteligencia artificial y la informática, utilizando principios de ingeniería de software basada en componentes, toma de decisiones distribuidas, computación paralela y distribuida, computación autónoma, métodos avanzados de interoperabilidad e integración de software.

La operación de un sistema basado en agentes se basa en interacciones de entidades de hardware o software autónomas y poco acopladas: agentes. Los procesos computacionales que se caracterizan por la descomposición natural o la posible distribución computacional pueden resolverse muy bien mediante sistemas de múltiples agentes. Además, el sistema de múltiples agentes ofrece una excelente capacidad de integración en tiempo de ejecución y una reconfiguración dinámica, y capacidades de delegación autónoma. Son robustos y proporcionan una fácil integración de humanos, software y hardware existentes (Franklin \& Grasesser, 2017).

La tecnología de agente se ha convertido recientemente en una de las áreas más vibrantes y de más rápido crecimiento en tecnología de la información. Los trabajadores involucrados en la investigación de agentes han ofrecido una variedad de definiciones. Estas definiciones van de lo simple a lo largo. Entonces podemos resumir todas estas definiciones en:

"El agente inteligente es un programa de software que realiza tareas para su propietario. Un agente recibe un conjunto de objetivos y luego actúa sobre su propietario para llevarlos a cabo. A medida que realiza 
sus tareas, aprende sobre su entorno, su propietario y otros agentes, y utiliza este conocimiento para mejorar cómo lleva a cabo sus tareas. Esta definición implica que las organizaciones de comunidades de agentes inteligentes brindan la oportunidad de desarrollar sistemas de apoyo a la toma de decisiones adaptables, para toda la organización e interorganizacionales, personalizados para usuarios específicos" (Mehmood, Ghafoor, Farooq, \& Zeeshan, 2018).

Un agente inteligente es un software que ayuda a las personas y actúa en su nombre. Los agentes inteligentes funcionan permitiendo que las personas deleguen el trabajo que hicieron en el software del agente. Los agentes pueden, solo datos complejos, aprender de usted e incluso hacerle recomendaciones.

Por lo tanto, los agentes de software se diferencian de otras aplicaciones por sus dimensiones adicionales de movilidad, autonomía, la capacidad de interactuar independientemente de supresencia del usuario y la capacidad de adaptación razonamiento. Esto implica la capacidad de procesar información de entornos externos, como redes, bases de datos e Internet, dado un conjunto de conocimientos, actitudes y creencias del usuario que el agente entiende. De la definición anterior, podemos deducir la característica clave del agente inteligente que la diferencia de otros tipos de aplicaciones de software:

1. Autonomía. El agente inteligente es capaz de actuar de forma independiente, exhibiendo control sobre su estado interno.

2. Reactividad. Un agente se Ilama reactivo cuando mantiene una interacción continua con su entorno y responde a los cambios que ocurren en él (a tiempo para que la respuesta sea útil).

3. Proactividad. Un agente es proactivo cuando es capaz de generar e intentar alcanzar objetivos; no impulsado única- mente por eventos; tomando la iniciativa

4. Habilidad social. En los agentes está la capacidad de interactuar con otros agentes (y posiblemente humanos) a través de algún tipo de lenguaje de comunicación de agentes, y tal vez cooperar con otros. A veces se le llama capacidad de comunicación del agente. Esto es para permitir que el agente inteligente tenga la información necesaria de diferentes fuentes.

5. Capacidad de cooperación: es el agente inteligente que coopera con otros agentes para el logro de ciertos objetivos.

6. Capacidad de razonamiento: es decir, el agente inteligente puede poseer la capacidad de inferir y extrapolar en función de los conocimientos y experiencias actuales.

7. Comportamiento adaptativo: es el agente inteligente que aprende o cambia su comportamiento en función de la experiencia previa.

8. Integridad: Es decir, el usuario debe tener una gran confianza en que sus agentes actuarán e informarán con sinceridad, y actuará por el bien del usuario. También hay otras propiedades de agencia a veces discutido:

- Movilidad: La capacidad de un agente para moverse por una red electrónica.

- Veracidad: Si un agente comunicará a sabiendas información falsa.

- Benevolencia: Si los agentes tienen objetivos en conflicto y, por lo tanto, si son inherentemente útiles.

- Racionalidad: Si un agente actuará para lograr sus objetivos, y no actuará deliberadamente para evitar que se logren sus objetivos.

- Aprendizaje / adaptación: Si los agentes mejoran el rendimiento con el tiempo.

No existe un diseño único de agente inteligente. No debemos suponer que todos los agentes inteligentes tendrán la misma co-

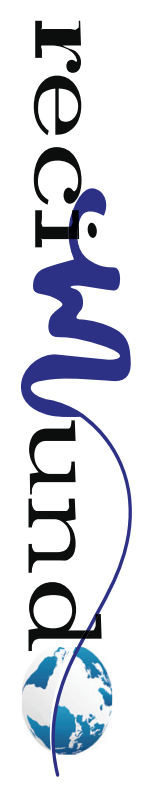


lección. Diferentes tipos de agentes pueden tener diferentes subconjuntos. Incluso entre los humanos hay una enorme diversidad, especialmente si consideramos casos extremos, como Newton, Mozart y sabios. Por lo tanto, no debemos suponer que un agente inteligente tiene una arquitectura fija: parte de los procesos de aprendizaje y desarrollo pueden incluir cambios en la arquitectura, por ejemplo, el desarrollo de nuevas colecciones importantes de capacidades y el desarrollo de nuevos vínculos entre las capacidades antiguas. Algunas personas parecen seguir desarrollando y ampliando sus arquitecturas por más tiempo que otras.

\section{Clasificaciones y aplicaciones de agen- tes}

Las diversas definiciones discutidas anteriormente involucran una serie de propiedades de un agente. Habiendo establecido una definición mucho menos restrictiva de un agente autónomo, estas propiedades pueden ayudarnos a clasificar aún más los agentes de maneras útiles. La siguiente imagen 1 enumera varias de las propiedades mencionadas anteriormente.

Cuadro 1. Propiedades de los Agentes

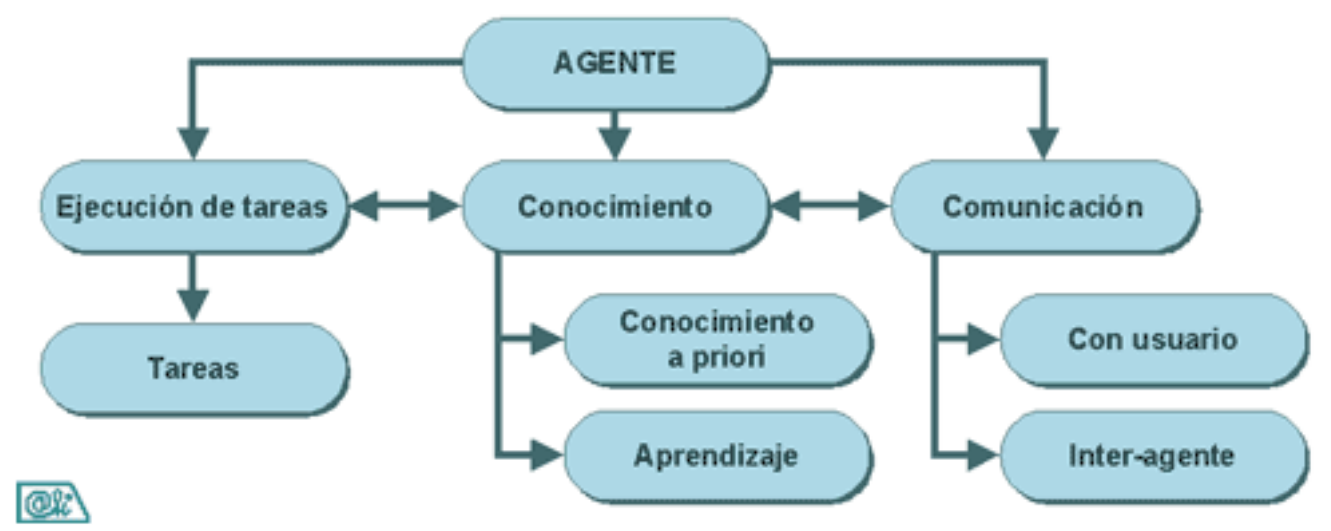

Fuente: (Inteligencia Artificial, 2020)

Los agentes pueden clasificarse útilmente de acuerdo con el subconjunto de estas propiedades que usan. Cada agente satisface las primeras cuatro propiedades. Agregar otras propiedades produce clases de agentes potencialmente útiles, por ejemplo, agentes de aprendizaje móviles.

Existen, por supuesto, otros posibles esquemas de clasificación. Por ejemplo, podríamos clasificar a los agentes de software de acuerdo con las tareas que realizan, por ejemplo, agentes de recopilación de información o agentes de filtrado de correo electrónico. O bien, podríamos clasificarlos de acuerdo con su arquitectura de control.
Un agente es un objeto de software que se comunica entre sí para realizar las tareas asignadas por su administrador. En un espectro más amplio, el agente trabaja en nombre de su usuario con objetivos y motivaciones similares. Un agente puede clasificarse según las funcionalidades de los siguientes tipos:

1. Agente autónomo: capaz de trabajar a través de una interfaz externa.

2. Agente adaptativo: responde en cierta medida a otro agente y entorno, y la adaptación permite que un agente modifique su comportamiento en función de su experiencia. 
3. Agente móvil: capaz de transportar de un entorno a otro.

4. Agente interactivo: se comunica con el entorno y otros agentes.

5. Agente proxy: puede actuar en nombre de alguien o algo.

6. Agente inteligente: el estado se formaliza mediante el conocimiento (es decir, creencias, objetivos, planes y suposiciones) e interactúa con otros agentes utilizando un lenguaje simbólico.

7. Agente coordinador: capaz de realizar alguna actividad en un entorno compartido con otros agentes.

8. Agente Cooperativo - Coordina con otros agentes para lograr un propósito común.

La taxonomía de los agentes de software de Brustoloni comienza con una clasificación de tres vías en agentes de regulación, agentes de planificación o agentes adaptativos (Ramos \& Quintero, 2008). Un agente de regulación probablemente nombrado con la regulación de la temperatura por un termostato o una regulación similar de la homeostasis corporal, reacciona a cada entrada sensorial a medida que entra y siempre sabe qué hacer. No planifica ni aprende. Los agentes de planificación planifican, ya sea en el sentido usual de IA (agente de resolución de problemas), o usando el paradigma basado en casos (agentes basados en casos), o usando métodos basados en investigación de operaciones (agentes OR), o usando varios algoritmos de aleatorización (agente aleatorio). Los agentes adaptativos de Brustoloni no solo planean, sino que aprenden. Por lo tanto, hay agentes de resolución de problemas adaptativos, y así sucesivamente, produciendo taxonomía de dos capas (Brustoloni, 1991).

Podemos tener una clasificación adicional del agente inteligente de la siguiente manera:

Supongamos que deseamos clasificar aún más a los agentes de software. ¿Cómo podríamos hacerlo? Los principales esquemas de subclasificación que vienen a la mente son las estructuras de control, los entornos (base de datos, sistema de archivos, red, Internet), el lenguaje (en el que está escrito) o las aplicaciones. Cada uno puede ser útil.

Primero, introduzcamos algunos de los posibles esquemas de clasificación inicial para los agentes de software a través de sus estructuras de control. (Brustoloni, 1991) ofrece regulación, planificación y adaptación. Otra estrategia se clasificaría por tipo de mecanismo de control, algorítmico, basado en reglas, difuso, red neuronal, aprendizaje automático, etc. O las clasificaciones binarias podrían ser planeación vs. no planificación, aprendizaje vs. no aprendizaje, móvil vs. no -móvil, comunicativo versus no comunicativo, etc.

Las tecnologías de los agentes son adecuadas para dominios que poseen cualquiera de las siguientes propiedades:

- Es necesario resolver problemas altamente complejos o controlar sistemas muy complejos.

- La información requerida para resolver problemas o controlar sistemas se distribuye y no está disponible centralmente,

- En dominios con entornos dinámicamente cambiantes y especificación de problemas.

- Es necesario integrar una gran cantidad de sistemas de software heterogéneos (y posiblemente hardware) de manera abierta y heterogénea.

- La cooperación de las unidades independientes (por ejemplo, en la organización virtual) está coordinada.

Las tecnologías de los agentes se adaptan a dominios que tienen una de las siguientes propiedades:

1. Requiere resolver problemas altamente complejos o controlar sistemas altamente complejos.

2. Ha distribuido información no disponible

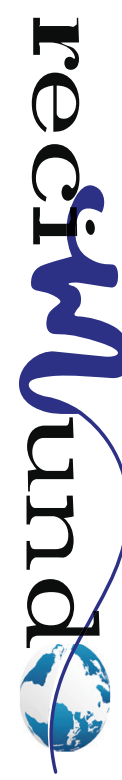


centralmente requerida para resolver problemas o controlar sistemas.

3. Tiene un entorno que cambia dinámicamente y especificaciones de problemas.

4. Debe integrar una gran cantidad de sistemas de software heterogéneos (y posiblemente hardware).

Se considera que los agentes como paradigma y las técnicas de agente disponibles funcionan bien en dominios de aplicación con ciertas propiedades específicas:

- Dominios competitivos y no cooperativos, donde las restricciones sobre el intercambio de información impiden el uso de la arquitectura centralizada de toma de decisiones, por ejemplo, aplicaciones de comercio electrónico, gestión de la cadena de suministro y comercio electrónico. En este caso, el paradigma de agente se utiliza para diseñar y describir los sistemas que actualmente están basados principalmente en la web.

- Dominios donde los datos requeridos para la toma de decisiones automatizada no están disponibles de forma centralizada debido a la distribución geográfica del conocimiento (p. ej., logística, exploración colaborativa, robótica móvil y colectiva, sistemas ubicuos) o los entornos con inaccesibilidad de comunicación parcial o temporal. Además de la distribución geográfica, también es distribución temporal (por ejemplo, en redes de satélite, donde los satélites tienen diferentes vistas de la tierra en diferentes momentos del día), y conceptual (por ejemplo, en jerarquías en capas, donde las entidades en una capa pueden no tener conocimiento de eventos o procesos en otras capas, como en Internet o en las cadenas de suministro)

- Dominios en los que se requiere una respuesta crítica de tiempo de supervivencia y una alta robustez en escenarios distribuidos. Este es el campo del control de sistemas industriales o de fabricación de tiempo crítico, donde se requiere una nueva planificación o una rápida reconfiguración local para manejar los problemas al instante.

- Dominios de simulación y modelado: El uso de agentes para fines de simulación ha sido muy común, mientras que a menudo faltaba la justificación correcta. Los agentes se pueden implementar en ejercicios de simulación en los que requerimos una migración fácil de la simulación al entorno real.

- Dominios con resolución de problemas complejos.: Esta ha sido la mayor expectativa de la tecnología de agentes en la industria. Un uso simple de técnicas de agente no puede resolver los problemas del complejo NP y el potencial de disminuir los requisitos computacionales para la resolución de problemas es limitado, pero posible.

- Sistema abierto La ingeniería ha sido enfatizada en los primeros proyectos, pero la realidad no cumple con las expectativas. Si bien las ontologías y los estándares FIPA han abordado muchos problemas de sintaxis, la semántica sigue siendo problemática. Los servicios web y las tecnologías web en general parecen liderar las aplicaciones en esta área.

Las tecnologías y diseños basados en agentes se han vuelto muy populares en el dominio de la defensa. Los proyectos exitosos en el dominio del comando y control, las transmisiones, la detección del entorno y la comunicación en el campo de batalla están en proceso de transformación desde la etapa de prototipo hacia futuras aplicaciones. Hay algunos ejemplos de la aplicación del agente en la vida real como sigue:

1. Actualmente, los sistemas de agentes se utilizan predominantemente en la gestión de producción orientada a proyectos donde ofrecen una ventaja competitiva significativa al adoptar fácilmente un entorno naturalmente muy dinámico. En este trabajo, estudiamos una aplicación 
de sistemas de múltiples agentes en una planta de fabricación altamente especializada y de gran volumen. La investigación subyacente es una extensión del proyecto de aplicación industrial real ejecutado para una importante planta de producción automotriz en Europa del Este.

2. Los aeropuertos para agentes 1 (AA) son una infraestructura distribuida de múltiples agentes implementada diseñada para un entorno de Internet dinámico e inestable. La infraestructura consta de plataformas llamadas Aeropuertos que permiten a los agentes comunicarse entre sí y ser persistentes. Además, los aeropuertos permiten a los agentes migrar a través del sistema y utilizar los recursos locales. Cualquier aeropuerto puede alojar a cualquier agente de la red; Por lo tanto, se considera requisitos altos para la seguridad (Rollo, Novak, \& Jisl, 2015). La red de aeropuertos puede cambiar dinámicamente en el tiempo a medida que los nuevos aeropuertos se conectan al sistema o se desconectan.

Varias áreas de aplicación de tecnología de agente se refieren típicamente a la fabricación. Pero, hay varias áreas de aplicación típicas de las tecnologías de agentes que se relacionan con la fabricación, como sigue:

1. En producción necesitamos resolver problemas de planificación altamente complejos; Necesitamos controlar procesos dinámicos, impredecibles e inestables. En la producción también existe la posibilidad de diagnóstico, reparación, reconfiguración y replanificación basados en agentes.

2. En el ámbito de las empresas virtuales y la gestión de la cadena de suministro, existen requisitos para formar alianzas comerciales, planificar acuerdos de cooperación a largo / corto plazo, gestionar (incluida la reconfiguración y disolución) de las cadenas de suministro. Aquí también podemos utilizar diversas tecnologías de agentes para el mantenimiento del conocimiento privado de los agentes, la especificación de diversas ontologías y garantizar la interoperabilidad del servicio en toda la cadena de suministro.

3. En el dominio de las tecnologías de agentes comerciales basadas en Internet, se pueden utilizar para compras y subastas inteligentes, recuperación y búsqueda de información, acceso remoto a la información y control remoto del sistema.

4. Un dominio de aplicación importante es la logística. Los sistemas de múltiples agentes se pueden utilizar para gestionar el transporte y la manipulación de materiales, la planificación y la programación óptimas, especialmente en el transporte de carga, el transporte público, pero también en misiones de mantenimiento de la paz, maniobras militares, etc.

5. Existe una buena combinación de las tecnologías de los agentes y la gestión de las redes de servicios públicos, tales como redes de distribución de energía, redes de operadores móviles, redes de proveedores de cable. Aquí, el concepto de computación autónoma distribuida se puede utilizar para la simulación y la predicción de situaciones de alarma, la prevención del apagón y la detección de sobrecarga e intrusión.

Los diferentes dominios de aplicación esperan que la tecnología del agente contribuya a diferentes clases de problemas. Se han identificado una lista de los malentendidos más comunes y las expectativas que el entusiasmo por la tecnología de los agentes puede causar:

1. Complejidad: A menudo se espera que la tecnología del agente pueda contribuir a resolver problemas muy complejos.

2. Caja negra: La tecnología de agente a menudo se ve como una tecnología de

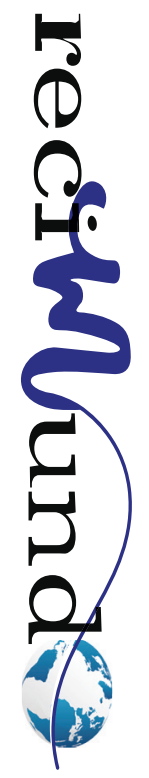


caja negra (como red neuronal, algoritmos genéticos) que se puede adaptar para resolver un problema complejo en particular. La tecnología de agente proporciona principalmente conceptos de sistema y paradigmas de diseño que son útiles en clases de problemas bien definidas.

3. Inteligencia: La resolución de problemas de los agentes, la inteligencia específica del dominio no es el problema estudiado (y se entrega) principalmente por la informática basada en agentes. La principal preocupación de los agentes investigadores es su comportamiento colectivo y la toma de decisiones, mientras que a menudo se pasa por alto la aplicación de la tecnología a problemas de la vida real.

4. Agentificación: El proceso de integración de agentes y la encapsulación de sistemas heredados se considera totalmente automatizado. No existe un mecanismo sofisticado que encapsule cualquier sistema heredado de manera totalmente automatizada. Las tecnologías alternativas (por ejemplo, servicios web) a menudo se utilizan en estos días.

5. Interoperabilidad: Los estándares y la interoperabilidad son computacionalmente caros. No es aconsejable utilizar el cumplimiento total de FIPA en un sistema donde la apertura total no es necesaria (por ejemplo, en simulación y modelado).

6. Aprendizaje: El potencial de aprendizaje en sistemas de múltiples agentes se sobreestima con frecuencia. A menudo se piensa que un agente debe ser súper adaptable y capaz de adaptarse a cualquier comportamiento solicitado (este comentario está estrechamente relacionado con los comentarios 3 y 4).

7. Movilidad: La movilidad del agente a menudo se afirma como más inevitable y esencial de lo que realmente se requiere. Muy a menudo, la migración de datos o la comunicación simple es suficiente en comparación con la migración del código y el estado del agente.

\section{Sistemas multi agentes}

La unidad de construcción básica en Multi Agent System (MAS) es un grupo de agentes. Los agentes inteligentes autónomos se comportan dirigidos por sus intenciones; Además, para alcanzar sus objetivos, pueden interactuar y cooperar con otros agentes. Esta interacción requiere que se les proporcionen capacidades comunicativas. Las claves de este sistema son la coordinación, cooperación y comunicación entre estos agentes (Wooldridge, 2012).

En un entorno distribuido, las aplicaciones y fuentes de administración de agentes deben coordinarse y cooperar entre sí para lograr un objetivo, esto se puede hacer utilizando uno de los métodos de comunicación. Se enfoca la intención a estas características importantes del sistema de múltiples agentes de la siguiente manera:

\section{1- Comunicación}

La comunicación es un concepto básico importante en Inteligencia Artificial Distribuida, porque es el proceso de interacción, a través de algunos tipos de comunicación, que hace posible que varios agentes combinen sus esfuerzos para resolver un problema general. La razón principal para la comunicación es resolver conflictos entre agentes.

Dado que los agentes actúan en un entorno que contiene otros agentes y diversos servicios, se necesita arquitectura para estandarizar la comunicación entre agentes y el acceso a servicios comunes. Los agentes pueden comunicarse enviando mensajes. Los mensajes pueden dirigirse a agentes individuales, a grupos de agentes o a todos los agentes de una clase en particular. La arquitectura de comunicación del sistema de agente debe proporcionar soporte para: comunicación de agente a agente, comunicación a través de una red y mecanismos 
de seguridad.

\section{2- Coordinación}

La coordinación es el proceso de manejar las interdependencias entre actividades. Para esto, varios agentes deben basar las comunicaciones en la detección y respuesta a las relaciones de coordinación existente entre las tareas conjuntas y conocidas.

La coordinación entre agentes se realiza solo cuando un agente puede comprender, o al menos predecir, las acciones de los otros agentes. Es decir, un agente debe tener algún modelo de otros agentes dentro de sí mismo. Para que un agente se adapte a la cooperación con otros, debe observar a otros agentes y establecer dinámicamente modelos de otros para planificar sus acciones.

Por lo tanto, cuando varios agentes intentan coordinar sus acciones, los agentes deben tener en cuenta las acciones potenciales de otros. Esto produce varios problemas complejos. Uno de los problemas es cómo un agente predice las próximas acciones de otros agentes en función de la información que ha adquirido.

Otro problema es cómo un agente planifica sus acciones teniendo en cuenta las acciones de los demás. Para predecir acciones de otros agentes, un agente debe poder modelar sus acciones dinámicamente. Para explicar cómo hacer un plan de sus acciones, un agente debe proporcionar estrategias y criterios efectivos para la cooperación.

\section{3- Cooperación}

Los agentes pueden ignorar, cooperar o competir entre sí. Lo más común es la cooperación de agentes donde los agentes combinan sus esfuerzos para lograr un objetivo común.
La mayoría de las interacciones entre agentes implican alguna forma de cooperación. En particular, a veces, los objetivos de un agente no se pueden lograr sin la ayuda de otros agentes. Si estos objetivos son comunes a un grupo de agentes (o un agente puede convencer a otros agentes para que lo ayuden), entonces se puede llegar a un acuerdo sobre un plan compartido. Una vez que se ha formado un grupo de agentes cooperantes, se produce un comportamiento especial relacionado con la necesidad de trabajar juntos hacia el objetivo común.

La cooperación entre los agentes incluye la noción de sobre ayuda, que se refiere explícitamente al reconocimiento de las intenciones de los otros agentes; Además, un agente puede adoptar y delegar a otros agentes para alcanzar objetivos de dominio y objetivos Meta, como planificar o controlar acciones. Las estrategias propuestas para la cooperación incluyen estrategias de cooperación fija, aprendizaje en trabajo en equipo, organizaciones jerárquicas, etc.

El sistema multiagente es la tecnología de inteligencia artificial distribuida. Esta tecnología se utiliza para vincular sistemas distribuidos lógica o geográficamente o para modelar la negociación en dichos sistemas. Diferentes requisitos pueden conducir a diferentes agentes. Para el entorno empresarial, el agente "es un agente de alguien" y desempeña cualquiera de los siguientes roles:

a) Asistente - la tarea del agente es adquirir y analizar información para apoyar la toma de decisiones del operador humano (socio).

b) Representante - Es una herramienta utilizada para la colaboración con otros. La negociación con un agente dentro del alcance de su poder es equivalente a la negociación con el socio.

c) Modelo - una representación simplificada de un compañero original (real) equipado

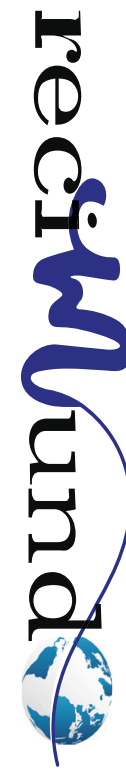


con los atributos y habilidades requeridos lo suficientemente acorde con los atributos y habilidades del compañero original. Para el dominio definido, el agente es un modelo del socio y puede actuar en nombre del socio emulando el comportamiento del socio.

Se puede ver que las funciones principales de los sistemas de múltiples agentes son:

1. Brindar continuidad y comunicación. protocolos entre los nodos para ayudar a predecir los resultados al extender el conocimiento especializado a otros dominios.

2. Integración y consistencia. del conocimiento debe proporcionarse automáticamente.

3. Romper los problemas complejos en un subproblema separado y proporcionando mediciones e interpretaciones por muchos expertos.

4. Cada agente puede aprender de los agentes de conocimiento si no resuelve algún problema.

Existen muchas soluciones prácticas diferentes y arquitecturas de sistemas de múltiples agentes. Por lo general, podemos identificar tres grandes grupos:

1. Sistema de múltiples agentes en el que ningún agente tiene conocimiento de otros miembros de la comunidad y tiene que usar la transmisión cuando quiera enviar cualquier información.

2. Sistema de múltiples agentes que utiliza un agente central (facilitador) que sirve como mediador.

3. Sistema multiagente con modelos sociales. que representan una solución de compromiso entre las dos primeras alternativas. Cada agente mantiene un modelo social de su entorno, modelos de comportamiento de los agentes cooperantes, su carga y disposición para cooperar.

\section{Taxonomía para sistemas multi agentes}

Un punto de partida para el desarrollo de estos principios es la definición de características básicas que definen el sistema inteligente de múltiples agentes. Extrapolando de las dimensiones de distribución, heterogeneidad y autonomía para el sistema de múltiples agentes, se pueden definir una serie de características generales:

A- Alcance del control funcional: una operación de agente individual en un sistema integrado puede tener un alcance de control que es (1) global, (2) local o (3) recursivamente local.

B- Enfoque operativo del sistema integrado: el sistema integrado puede acceder a agentes individuales a través de (1) funciones locales, (2) interfaces locales o (3) parámetros de aplicación o problemas.

C- Locus de control del sistema: el sistema de resolución de problemas puede tener un locus de control definido recursivamente que varía de (1) centralizado a (2) descentralizado.

D- Mecanismo de integración del sistema: el mecanismo de integración del sistema puede basarse en (1) funciones, (2) lenguajes, (3) esquemas de representación o (4) aplicaciones o problemas.

E- Composición de los agentes: los agentes pueden ser (1) homogéneos o (2) heterogéneos.

F- Tipos de agentes del sistema: los agentes que comprenden el sistema integrado pueden ser (1) humanos, (2) máquinas, o (3) composiciones de humanos y máquinas.

El sistema actual de múltiples agentes (independientemente de su dominio) se está volviendo complejo y, por lo tanto, difícil de evaluar y analizar. Esto es debido a: 
- Agentes cada vez más complejos con muchas propiedades dinámicas.

- El sistema de múltiples agentes consta de muchos objetos y agentes.

- Los agentes suelen tener arquitecturas diversas, no muy transparentes.

- Muchos dominios de implementación posibles (diferentes actuadores y sensores, etc.)

- El entorno se está volviendo más complejo y varía en el tiempo.

Hay algunas razones por las cuales las personas usan un sistema de múltiples agentes para el modelado y la simulación (a diferencia de las técnicas clásicas de modelado matemático y simbólico) es una transición fácil y natural del proceso de modelado a la operación real distribuida. Para la operación distribuida (por ejemplo, control, diagnóstico, planificación ...) se puede implementar el mismo conjunto de agentes que se utilizan en la fase de modelado. Los socios industriales a menudo requieren pruebas sustanciales y verificación experimental de los sistemas avanzados de IA antes de que estén listos para el despliegue industrial. La tecnología de agente es un concepto ideal para esto (Platon, Rehak, \& Sabouret, 2017).

\section{Conclusiones}

Los sistemas de múltiples agentes tiene como objetivo lograr un comportamiento de conmutación por error robusto y confiable mediante apertura, reconfiguración del tiempo de ejecución, replanificación dinámica y selección de socios y mayor autonomía del agente en general. Por lo tanto, su aplicación puede mejorar significativamente la confiabilidad del sistema cuando encuentra fallas aleatorias, por ejemplo, mal funcionamiento del hardware, problemas de comunicación, fallas en la batería / fuentes de alimentación y otros.

Y, la experimentación en la vida real puede ser costosa, tomar mucho tiempo o incluso ser imposible debido a razones físicas o de seguridad. Una de las formas de lidiar con este problema es crear un modelo del proceso o entorno de la vida real y usar una simulación de software.

Los sistemas de agentes múltiples son una opción natural para modelar sistemas distribuidos que consisten en entidades autónomas, interesadas en sí mismas, como los equipos de robots autónomos. Dicho modelo puede usarse con ventajas para simular y analizar aspectos específicos del comportamiento general del sistema, como la coordinación de actividades, la negociación o la comunicación mutua. Además de esto, el modelo también se puede utilizar para desarrollar y probar algoritmos de control antes de su implementación práctica.

\section{Bibliografía}

Boella, G., Damiano, R., \& Lesmo, L. (2009). A Utility Based Approach to Cooperation among Agents. New York: McGraw Hill.

Bolívar, J. (2015). Investigación Documental. México: Pax.

Brustoloni, J. (1991). Autonomous agents: Characterization and requirements. School of Computer Science, Carnegie Mellon University.

Castro, J. (2016). Técnicas Documentales. México. Limusa.

Davila, A. (2015). Concepto de terminos cientificos. Caracas: Oasis.

Elmahalawy, A. (2011). Utilization of Multi-Intelligent Agent Systems for Problem Solving. M.Sc. Thesis, Egypt: Menofia University, Faculty of Electronic Engineering, Department of Computer Science and Engineering.

Franklin, S., \& Grasesser, A. (2017). Is it an Agent or just a program?: A Taxonomy for Autonomous Agents. Third International Workshop on Agent Theories, Architectures, and Languages, 21-35.

Inteligencia Artificial. (20 de 02 de 2020). AGENTES INTELIGENTES. Obtenido de https://sitiointeligenciaa.wordpress.com/agentes/

Mehmood, A., Ghafoor, A., Farooq, A., \& Zeeshan, I. (2018). Adaptive Transport Protocols in Multi Agent System. Fifth International Conference on Information Technology, 720-725. 
Platon, E., Rehak, M., \& Sabouret, N. (2017). Detecting Intrusions in Agent System by Means of Exception Handling. HoloMAS, 161-172.

Ramos, A., \& Quintero, A. (2008). Model of Coordination of Multi-Agent Systems Applied to Who Accomplish Labor of Auditory of Systems of an Organization. Orlando, Florida: H. and Olson, R. .
Rollo, M., Novak, P., \& Jisl, P. (2015). Simulation of Underwater Surveillance by a Team of Autonomous Robots. HoloMAS, 207-220.

Wooldridge, M. (2012). An Introduction to Multi-Agent Systems. Johen Wiely\&Sons.

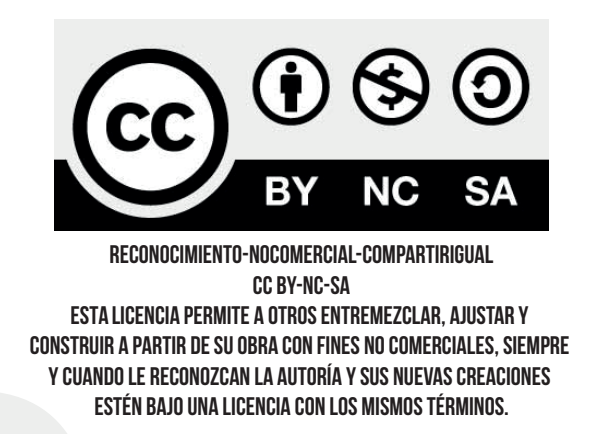

\section{CITAR ESTE ARTICULO:}

Coloma Garofalo, J., Vargas Salazar, J., Sanaguano Guevara, C., \& Rochina Chisag, Á. (2020). Inteligencia artificial, sistemas inteligentes, agentes inteligentes. RECIMUNDO, 4(2), 16-30. doi:10.26820/recimundo/4.(2). mayo.2020.16-30 